\title{
Criminologie
}

\section{Plaidoyer pour la sociologie criminelle}

\section{Pierre Tremblay}

Volume 22, numéro 2, 1989

Le milieu criminel

URI : https://id.erudit.org/iderudit/017279ar

DOI : https://doi.org/10.7202/017279ar

Aller au sommaire du numéro

Éditeur(s)

Les Presses de l'Université de Montréal

ISSN

0316-0041 (imprimé)

1492-1367 (numérique)

Découvrir la revue

Citer cet article

Tremblay, P. (1989). Plaidoyer pour la sociologie criminelle. Criminologie, 22(2), 5-12. https://doi.org/10.7202/017279ar d'utilisation que vous pouvez consulter en ligne.

https://apropos.erudit.org/fr/usagers/politique-dutilisation/ 
Cette section thématique de la revue Criminologie apparaîtra, je crois, passablement inhabituelle, à la fois par son contenu et par sa forme. Les articles qui y sont présentés sont tous consacrés au milieu criminel, un objet d'étude que la sociologie criminelle a le plus souvent délaissé et qu'elle maîtrise, aujourd'hui encore, fort mal. Par ailleurs, la plupart de ces articles n'ont pas été rédigés par des chercheurs travaillant de façon indépendante sur le même sujet, mais par un groupe de recherche (rattaché au Centre international de criminologie comparée), pratiquant sur le même terrain (en l'occurrence celui du milieu criminel montréalais) et dont les travaux souvent se recoupent à la fois empiriquement et théoriquement. Quelques remarques générales sur les difficultés qu'ont rencontrées les sociologues qui se sont intéressés au milieu criminel et sur la manière dont ce groupe de recherche a tenté de les résoudre me semblent donc utiles et elles permettront peut-être de mieux comprendre le contexte dans lequel ces travaux ont été réalisés.

\section{L'ÉCOLE DE CHICAGO}

Pour fins d'analyse, nous définirons le milieu criminel comme l'ensemble des interactions directes ou indirectes entre individus ayant choisi de participer de façon relativement régulière à un ensemble variable d'activités criminelles. Le milieu criminel désigne donc toujours par définition un sous-ensemble de la population des délinquants. La seule tentative systématique d'étudier le milieu criminel date des années 1920-1930 et on la doit à un groupe de sociologues que l'on a par la suite identifié comme faisant partie de l'École de Chicago (Downes et Rock, 1986; Chapoulie, 1984; Snodgrass, 1972). La contribution de l'École de Chicago à l'étude des milieux criminels mérite d'être soulignée pour deux raisons: la première peut être qualifiée de nostalgique. Les seules personnes pour qui l'actualité des chroniques policières ou judiciaires trouve encore des résonances particulières sont les journalistes qui y sont assignés, les policiers, les délinquants qui participent au milieu criminel et les gardiens de prison. La sociologie criminelle contemporaine s'est largement dissociée de ce savoir atomisé et informel. Mais cela n'a pas toujours été le cas. L'École de Chicago a mérité son appellation non pas parce que les chercheurs qui y ont participé enseignaient dans cette ville mais parce

*Professeur-adjoint, Département de sociologie, Université McGill et chercheur associê au Centre international de criminologie comparée de l'Université de Montréal. 
que leur objet d'étude était la ville de Chicago elle-même et qu'ils entreprirent, à la façon des ethnologues ou des anthropologues, d'observer sur le terrain non seulement les bas-fonds de Chicago mais la diversité des micro-sociétés qui s'y côtoyaient. Une quantité assez impressionnante de classiques mineurs furent publiés dans les années 1920-1930, chaque étude se présentant comme une monographie détaillée sur une collectivité ou un groupe social particulier: les bandes de délinquants juvéniles, le milieu de la pègre, les réseaux de prostitution commerciale, les dancings, le monde des clochards et des chambreurs, l'univers des agents immobiliers ou celui des communautés ethniques. Il ne fait pas de doute que ces sociologues connaissaient Chicago et que leur longue fréquentation des classes laborieuses et des classes dangereuses faisait en sorte que l'actualité des chroniques judiciaires et policières non seulement leur disait quelque chose mais avait des résonances et que ces résonances alimentaient à leur tour leurs propres travaux. Une des raisons pour lesquelles la sociologie criminelle maîtrise mal l'analyse du milieu criminel tient, je crois, à son incapacité d'interpréter les faits divers, et cette ignorance résulte, comme on le verra, de son manque d'information quant à la façon dont les crimes sont pratiqués.

La deuxième raison est plus théorique. On a retenu surtout des travaux de l'École de Chicago, les études de géographie criminelle. On s'est peu attardé cependant aux diverses raisons pour lesquelles les sociologues de l'École de Chicago se préoccupèrent au départ des zones urbaines criminelles, de leur genèse, de leur stabilité et de leur localisation. C'est qu'elles représentaient pour eux la manière la plus simple et la plus riche d'analyser les interactions entre délinquants, et plus spécifiquement les échanges et interactions qui résultaient de leur concentration dans certains quartiers ou ghettos. Nous proposons dans ce numéro une généralisation et un léger déplacement de point de vue. L'analyse du milieu criminel peut être élaborée, et de manière plus systématique, en mettant l'accent non plus sur les interactions entre délinquants, résultant de leur concentration dans des quartiers particuliers, mais sur les échanges et les conflits résultant de leur participation à des activités criminelles spécifiques, celles-ci pouvant ou non être territorialisées dans certains quartiers urbains. Une telle analyse ne pouvait pas être entreprise à l'époque de l'École de Chicago pour une raison fort simple: les corps de police des années 1920 n'étaient ni bureaucratisés ni informatisés et les unités d'enquêtes spécialisées que nous connaissons aujourd'hui n'existaient que sous une forme embryonnaire. (Sur ce thème voir Tremblay et Kedzior, 1985). Comme le mentionne 
Reuter (1983), la seule étude sur la pègre de l'époque, celle de F. Landesco, était basée sur les renseignements recueillis par un groupe de pression communautaire et civique. Dans la mesure où l'analyse du milieu criminel au sens où nous l'entendons ici présuppose le traitement de ce que nous appelons les archives policières, les facilités matérielles que requiert le genre d'études que nous présentons dans ce numéro n'existaient pas.

\section{PRINCIPES D'ANALYSE DU MILIEU CRIMINEL}

Une des difficultés que pose l'étude des milieux criminels réside dans le fait que les règles élémentaires de méthode doivent être découvertes et éclaircies au fur et à mesure et qu'il n'existe aucune tradition de recherche dans ce domaine. (Voir cependant P. Reuter, 1983, p. 188197). Voici quatre principes d'analyse qui nous semblent donner à ce genre d'étude sa configuration spécifique:

\section{A) RECONSTITUER DES POPULATIONS COMPLÈTES}

Alors que les sociologues procèdent le plus souvent par sondage ou par entrevue individuelle, l'analyse du milieu criminel a généralement la tâche de reconstituer des «populations» complètes, (quoique très singularisées) d'acteurs. Non pas un fraudeur ou un tueur ou un voleur, non pas non plus un échantillon de fraudeurs ou de cambrioleurs, mais la population complète des fraudeurs d'un certain genre, des tueurs d'un certain type, dans un contexte social déterminé. Dans l'article de Yves Leguerrier sur la prostitution, c'est la population complète des studios de massage et des agences d'escorte ayant opéré dans la région métropolitaine de Montréal au début des années 1980 qui est reconstituée. Dans l'article que Gilbert Cordeau consacre aux règlements de comptes, c'est la population complète des individus directement impliqués dans les règlements de comptes commis dans la province de Québec durant les dernières 20 années qui est analysée. Dans une étude similaire consacrée aux fraudes par carte de crédit, c'est la population complète de ceux qui participèrent à une fraude bancaire très particulière qui démarra à Montréal en décembre 1980 pour se terminer en avril 1981 que j'ai essayé de retrouver (Tremblay, 1986). Spécification contextuelle très fine et reconstitution de populations complètes d'individus constituent les premières caractéristiques de l'analyse du milieu criminel soulevant un ensemble de problèmes méthodologiques qui lui sont propres. 


\section{B) ANALYSER LES PRATIQUES CRIMINELLES}

Il est surprenant que la sociologie criminelle ait choisi pendant si longtemps d'ignorer théoriquement la manière dont les activités criminelles se pratiquent concrètement (pour un constat similaire, voir Cusson, 1981 ou Clarke et Cornish, 1985). Il est impossible d'entreprendre une analyse du milieu criminel sans connaître en détail la manière dont se pratique une variété importante d'activités criminelles susceptibles d'attirer, dans un contexte social ou urbain donné, la majeure partie de ceux qui y participent. En termes pratiques, et par expérience, il nous est apparu très difficile de comprendre (au sens weberien du crime, cf. Boudon, 1985) une pratique criminelle en moins d'un an. Il est impossible, par exemple, de comprendre les braquages de banque sans une analyse détaillée de la manière dont les braqueurs se procurent leurs armes et une évaluation de l'ampleur et du fonctionnement du marché noir des armes à feu de petit calibre. Et parce que bon nombre de braqueurs opèrent en volant la veille ou le jour même le véhicule dont ils se servent pour prendre la fuite, une analyse de l'impact des vols qualifiés sur le taux de véhicules volés doit être prévue dans ce genre de recherche. Et dans la mesure où le braquage de banque ne constitue pour ceux qui s'y livrent qu'une des opportunités criminelles qui leur sont accessibles, les contingences particulières qui font fluctuer le taux de braquage méritent également d'être examinées avant que l'on puisse prétendre comprendre la manière dont il se pratique ou la fréquence optimale de son exécution. L'analyse que Yves Leguerrier propose du marché de la prostitution commerciale satisfait de manière générale à ces critères précisément parce qu'il s'est attardé (comme le suggéraient Clarke et Cornish, 1985), à l'ensemble des décisions que doivent prendre les entrepreneurs qui participent à ce marché s'ils veulent espérer pouvoir y survivre plus que quelques semaines. Mais la raison pour laquelle l'analyse du milieu criminel présuppose la reconstitution stratégique (selon l'expression de Cusson, 1986) des pratiques criminelles tient au fait que celles-ci nous font découvrir le plus souvent, et «en prime», jusqu'à quel point et de quelle façon les structures d'opportunités criminelles sont étroitement imbriquées les unes aux autres et déterminent, peut-être, en dernière instance, la trajectoire des carrières criminelles individuelles.

\section{C) COMPARER LES STRUCTURES D'OPPORTUNITÉS CRIMINELLES}

On ne peut comprendre le fonctionnement d'une variété particulière de fraude par carte de crédit sans reconstituer la famille des quatre sortes de fraudes «au volume» pratiquées par le milieu des fraudeurs à 
Montréal (Tremblay, 1986), et sans prendre en considération celles qui n'opèrent pas au volume. L'étude que Yves Leguerrier nous propose sur la prostitution commerciale présente une analyse comparative systématique de deux marchés bien distincts de prostitution (studios de massage vs agences d'escorte). L'intérêt de l'analyse par Gilbert Cordeau des règlements de comptes tient à sa classification des trois sortes de conflits endogènes au milieu criminel. L'analyse d'étude (encore inédite) d'un marché de paris illicites (associé à l'exploitation des appareils d'amusement de type bingo ou poker) fait usage d'une analyse comparée du profil typique des carrières criminelles caractéristiques de diverses catégories d'acteurs du milieu criminel (Tremblay, Cordeau, Leguerrier, 1989). Et l'article consacré aux motards activement impliqués dans le milieu utilise la banque de données sur les règlements de comptes mise au point par Gilbert Cordeau afin de spécifier le sousensemble des homicides imputables aux motards eux-mêmes et de déterminer par la suite jusqu’à quel point ceux-ci méritent leur réputation de violence. Recoupements et analyses comparées sont essentiels à toute analyse du milieu criminel pour deux raisons. Tout d'abord, la plupart de ceux qui pratiquent le crime sur une base relativement régulière font toujours face à un éventail d'opportunités criminelles et le problème n'est pas tant celui de choisir entre le crime et le non-crime (un problème qui intéresse la sociologie criminelle classique) mais de choisir entre diverses sortes d'activités illicites, chacune ayant ses avantages et ses inconvénients. Deuxièmement, les marchés criminels et plus généralement les structures d'opportunités criminelles sont elles-mêmes couplées et inter-reliées et dans une large mesure interdépendantes, de sorte qu'une innovation ou un changement local peut avoir des effets indirects importants sur les conditions de fonctionnement de l'ensemble du milieu. Si l'analyse comparative semble aller de soi, un examen rapide de la sociologie criminelle classique suggère que tel n'est pourtant pas le cas : la plupart des études analysent des catégories abstraites de crimes plutôt que des pratiques criminelles, et lorsque les travaux se concentrent sur une forme d'activité criminelle, celle-ci est analysée indépendamment des autres formes d'opportunités criminelles connexes (Cornish et Clarke, 1989, font un constat similaire).

\section{D) ANCRER LES STRUCTURES D'OPPORTUNITÊ CRIMINELLE}

Une demière caractéristique des analyses du milieu criminel présentées dans ce numéro tient à la manière dont elles font un usage systématique de sources documentaires multiples. La banque de données sur les règlements de compte reconstituée par Gilbert Cordeau fait 
usage non seulement des dossiers d'enquête des escouades sur l'homicide de deux corps de police mais procède aussi à l'analyse de contenu systématique d'un hebdomadaire spécialisé dans les affaires d'homicide (Allô Police). L'étude d'Yves Leguerrier sur le marché commercial de la prostitution se base à la fois sur les annonces classées des quotidiens et sur les dossiers des escouades de moralité de la région métropolitaine montréalaise. L'analyse de l'industrie des appareils d'amusement fait usage non seulement des dossiers d'enquête en matière de jeux et paris mais d'un sondage sur le terrain auprès des entreprises qui se sont enregistrées à la Régie des loteries et courses du Québec. La raison d'être de ces sources documentaires multiples n'est pas simplement méthodologique et n'a pas pour seule fonction d'asseoir par corroboration ou triangulation la validité des renseignements recueillis. Comme le soulignent, de manière générale, Felson et Cohen (1980), les opportunités criminelles se nichent et s'intègrent étroitement dans les structures d'opportunités légitimes, de sorte qu'il n'est pas étonnant que l'analyse des marchés criminels débouche souvent sur la manière dont ceux-ci parasitent une infra-structure sociale ou économique parfaitement légitime. Certaines fraudes par carte de crédit exploitent les stratégies de marketing des banques (Tremblay, 1986) et les travaux en cours (non publiés) que Leguerrier consacre au recel des véhicules suggèrent que le marché noir du recel dépend étroitement des caractéristiques potentiellement criminogènes de l'industrie automobile ellemême (Faberman, 1975). Alors que l'École de Chicago s'intéressait aux zones sociales désorganisées propices au développement de traditions criminelles, l'analyse du milieu criminel s'intéresse plutôt à la manière dont les opportunités criminelles que ce milieu exploite ou génère s'intègrent au tissu social. Dans le premier cas on s'intéresse aux raisons pour lesquelles on devient criminel et dans le second, à la manière dont il est effectivement possible et intéressant de le demeurer.

\section{CONCLUSION}

L'analyse des pratiques criminelles présuppose une sorte de contrat implicite entre chercheurs et corps policiers. Comme l'a déjà souligné Brodeur (1983) ce contrat s'est jusqu'à présent révélé hautement problématique ainsi qu'en témoignent les polémiques entourant la publication récente des travaux d'Ericson $(1981,1982)$ sur les patrouilleurs et les enquêteurs de Toronto. Une des raisons pour lesquelles les modalités implicites de collaboration entre chercheurs et autorités policières ont toujours été compliquées tient en partie, à mon avis, à un double quiproquo. L'ignorance des criminologues ou des 
sociologues en matière de pratiques criminelles, combinée à une politisation excessive de leurs préoccupations, les a convaincus que leur champ d'étude devait porter sur la police elle-même plutôt que sur sa raison d'être (la criminalité en général mais plus particulièrement le sous-ensemble le plus actif et le plus constant des délinquants). À l'inverse, les autorités policières ont depuis longtemps été convaincues, et à juste titre souvent, de détenir le monopole de la compétence en matière de criminalistique ou de connaissance des pratiques criminelles, de sorte que l'utilité des civils ne pouvait logiquement se justifier que dans la mesure où ceux-ci s'intéressaient moins à la sociologie criminelle fondamentale qu'à l'évaluation critique de leurs manières d'opérer. Toutefois, l'informatisation des banques de données policières, la spécialisation et la bureaucratisation des unités d'enquête et le développement des services de renseignements ont fait en sorte que les principaux corps policiers disposent actuellement (contrairement à la situation qui prévalait il y a une dizaine d'années) d'une masse documentaire suffisamment organisée pour servir réellement de laboratoire quasi expérimental à une sociologie criminelle en manque de raffinements théoriques. L'institutionnalisation d'un tel labo (une sorte d'unité d'analyse stratégique des renseignements criminels) serait d'autant plus souhaitable que les états-majors éprouvent une difficulté croissante à maîtriser, à des fins autres que purement tactiques ou ponctuelles, le volume considérable d'informations dont ils disposent.

\section{RÉFÉRENCES}

BOUDON, R. (1985), La place du désordre: critiques des théories du changement social, Presses universitaires de France, Paris.

BRODEUR, J.P. (1983), Production du crime et reproduction de l'ordre selon Ericson : quelques réflexions critiques, Revue canadienne de criminologie, 25, 1, 105-118.

CHAPOULIE, J.-M. (1984), Everett C. Hughes et le développement du travail de terrain en sociologie, Revue française sociologique, 25, 582-608.

CLARKE, R.V.G., CORNISH, D.B. (1985), Modeling Offender's Decision: A Framework for Research and Policy, in M. Tonry, N. Morris (édit.) Crime and Justice, vol. 6, Chicago : University of Chicago.

CORNISH, D.B., CLARKE, R.V.G. (1989), Crime Specialisation, Crime Displacement and Rational Choice Theory, in $\mathrm{H}$. Wegener, F. Losek, J. Haish (édit.) Criminal Behavior and the Justice System : Psychological Perspectives, New York : SpringerVerlag, (sous presse).

CUSSON, M. (1981), Délinquants pourquoi? Paris : Armand Collin.

CUSSON, M. (1986), L'analyse stratégique et quelques développements récents en criminologie, Criminologie, 19, 1, 53-72. 
DOWNES, D.M., ROCK, P. (1986), Understanding Deviance : A Guide to the Sociology of Crime and Rule-Breaving, Toronto : Oxford University Press.

ERICSON, R.V. (1981), Making Crime: A Study of Detective Work, Toronto; Butterworths.

ERICSON, R.V. (1982), Reproducing Order: A Study of Police Patrol Work, Toronto: University of Toronto Press.

FABERMAN, H.A. (1975), A Criminogenic Market Structure : The Automobile Industry, Sociological Quarterly, 16, 3, 438-57.

FELSON, M., COHEN, L.E. (1980), Human Ecology and Crime: A Routine Activity Approach, Human Ecology, 8, 4, 389-406.

LANDESCO, J. (1968), Organized Crime in Chicago, Chicago : University of Chicago Press.

REUTER, P. (1983), Disorganized Crime: The Economics of the Visible Hand. Cambridge, MIT Press.

SNODGRASS, J. (1972), The American Criminological Tradition : Portraits of the Man and Ideology in a Discipline, thèse de doctorat, University of Pennsylvania.

TREMBLAY, P., KEDZIOR, R. (1985), Notes introductives à l'analyse du crime organisé contemporain: prolégomènes montréalais, Revue canadienne de criminologie, 27, 2, 179-194.

TREMBLAY, P. (1986), Designing Crime : The Short Life Expectancy and the Workings of a Recent Wave of Credit Card Bank Fraud, British Journal of Criminology, 26, 3, 234-253.

TREMBLAY, P., CORDEAU, G., LEGUERRIER, Y. (1989), Un marché de paris illicites: une étude de cas, Centre international de criminologie comparée, Université de Montréal, 37 pages. 